Revista General de Información y Documentación ISSN: 1132-1873

http://dx.doi.org/10.5209/RGID.60814

\title{
Depósito Legal en línea: desafíos para su desarrollo en España
}

Lourdes Santos de Paz ${ }^{1}$

Recibido: 31 de octubre 2017 / Aceptado: 9 de febrero de 2018

Resumen. El estudio pretende mostrar los retos a los que se enfrentan las instituciones conservadoras y las estrategias que se deben plantear para llevar a cabo la preservación del patrimonio cultural que se produce de manera intangible en el territorio español. La necesidad de preservar los documentos producidos actualmente de forma intangible ha provocado un cambio en el proceso de gestión del Depósito Legal de manera que las instituciones de custodia han de afrontar nuevos desafíos para que en España se lleve a cabo el desarrollo necesario que ha comenzado a regularse a partir del Real Decreto 635/2015 de 10 de julio que viene a desarrollar la Ley 23/2011 de Depósito Legal.

Palabras clave: Archivado de la web; Colecciones digitales, Depósito Legal en línea; Patrimonio digital español; Preservación digital.

\section{[en] Deposit-online: challenges for it development in Spain}

\begin{abstract}
The study aims to show the challenges facing by conservative institutions and the strategies that must be proposed to carry out the preservation of the cultural heritage that is produced in an intangible way in the Spanish territory. The need to preserve the documents currently produced in an intangible way has caused a change in the process of management of the Legal Deposit so that custody institutions have to face new challenges in order to carry out the necessary development in Spain regarding legislation from the Royal Decree 635/2015 of 10 July that comes to develop Law 23/2011 of Legal Deposit.
\end{abstract}

Keywords: Archiving of the web; Digital collections; Digital preservation; Online Legal Deposit; Spanish digital Heritage.

Sumario. 1. Introducción; 2. Problemática del documento electrónico; 3. Modelos en otros países; 4. Los conservadores de la web en España; 5. Conclusiones; 6. Referencias bibliográficas.

Cómo citar: Santos de Paz, L. (2018) Depósito Legal -en línea: desafíos para su desarrollo en España Legal, en Revista General de Información y Documentación 28 (1), 11-22.

1 Universidad de León. Biblioteconomía y Documentación

E-mail: lourdes.santos@unileon.es 


\section{Introducción}

Las nuevas formas de comunicar y generar conocimiento están produciendo la información de una manera intangible que es necesario gestionar y preservar para las generaciones futuras. La idea promovida por la UNESCO, organización que vela por la herencia de la identidad y la memoria culturales, es que el patrimonio digital susceptible de ser preservado se ha de formar con el conjunto de "recursos de carácter cultural, educativo, científico o administrativo e información técnica, jurídica, médica y de otras clases, que se generan directamente en formato digital o se convierten a éste a partir de material analógico ya existente”. (Unesco, 2003).

Históricamente la misión del Depósito Legal ha residido en recoger y conservar para preservar todo el patrimonio bibliográfico y documental de un país. Actualmente se producen una serie de circunstancias que añaden cambios a este proceso a causa de las nuevas formas de comunicar y generar conocimiento, esto es, la producción de información en línea o digital.

En el caso de España, en 2015 ha visto la luz el Real Decreto que regula el Depósito Legal de las publicaciones en línea (DL-e), tan necesario como esperado, que sistematiza la incorporación a la custodia para el futuro, de esa actualidad informativa producida en Internet o en forma digital, de la que España no puede ausentarse. El Real Decreto aprobado en Consejo de Ministros el 10 de julio de 2015, no es más que un desarrollo imprescindible de la Ley 23/2011, de 29 de julio de Depósito legal que ya ampliaba, en un intento de modernización, la necesidad de proporcionar mecanismos para recoger aquellos documentos en línea y digitales que se reconocen como parte del patrimonio bibliográfico y documental. ${ }^{2}$

Los documentos electrónicos recogidos por Depósito legal abren un horizonte nuevo frente a la manera en que se venía recogiendo toda la producción bibliográfica hasta el momento actual. El escenario ofrece hoy una convivencia de sistemas, de tal modo que el ingreso de los todavía documentos tangibles comparte proceso con la recogida de los libros electrónicos. Ambos llevan cauces completamente diferentes. Por ejemplo la iniciativa de depósito de algunos de estos documentos electrónicos ya no está en los editores sino que serán los conservadores web los que decidan qué pasará a integrar el archivo de preservación para el futuro, sin bien en el caso de libros electrónicos, el productor deberá facilitar el acceso para la captura.

Este estudio pretende mostrar los retos a los que se enfrentan las instituciones conservadoras y las estrategias que se deben plantear para llevar a cabo la preservación del patrimonio cultural que se produce de manera intangible en el territorio español.

2 Nos basaremos en este decreto, en los informes del Grupo de trabajo de Depósito Legal y Patrimonio digital además de la consulta realizada al Manual para conservadores web realizado por el área de Gestión del Depósito de publicaciones en línea de la BNE. 


\section{Problemática del documento electrónico}

El proceso de Depósito legal para los documentos impresos lleva mucho tiempo consolidado y no reviste cambios, sin embargo el método para recoger los documentos en línea que, amparados en la Ley 23/2011 es de obligado cumplimiento depositar para reunir y completar el patrimonio documental que se produce en soportes nuevos, se muestra inicialmente complejo. Estos documentos sin equivalencia en el mundo analógico, denominados born digital, así creados (Serra, 2006), requieren unas condiciones explícitas de funcionalidad, originalidad y autenticidad. Algo que entra en conflicto con la forma de editar puesto que se desarrolla en un entorno cambiante, basándose en una tecnología competitiva en permanente evolución. Ciertamente se trata de aspectos que otorgan una dificultad inicial en el compromiso por garantizar la preservación y el acceso a largo plazo.

Es evidente, por otro lado, que la producción de información en modo digital crece de una manera desorbitada, lo que plantea cierta complejidad en su gestión y conservación a las instituciones encargadas de su custodia. Conviene reseñar que la facilidad y el abaratamiento de los costes de publicación unidos a la obligación de preservar lo producido a la vez que darle accesibilidad, son circunstancias que empujan a los centros responsables de la custodia a utilizar nuevas metodologías que les permitan adecuarse de forma inminente al proceso. Las instituciones de la memoria, entre las que se cuentan archivos y bibliotecas, tienen ante sí el reto de garantizar el acceso permanente al patrimonio documental digital. Significa que se debe actualizar el procedimiento que permita llevar a cabo las obligaciones de recoger, gestionar, preservar lo digital que forma parte del Depósito legal (De Beer, et al. 2015).

\subsection{Características de los documentos electrónicos}

Como bien se señala en la Carta de la Unesco (2003) : los objetos digitales pueden ser textos, bases de datos, imágenes fijas o en movimiento, grabaciones sonoras, material gráfico, programas informáticos o páginas web, entre otros muchos formatos posibles dentro de un vasto repertorio de diversidad creciente. A menudo son efímeros, y su conservación requiere un trabajo específico en este sentido en los procesos de producción, mantenimiento y gestión.

Si buscamos en el decreto referido, los criterios que regirán para seleccionar los archivos de la web aparecen mínimamente establecidos, a pesar de que se obliga a recoger por Depósito legal (art. 3) todo tipo de sitios web y publicaciones en ellos integradas siempre que contengan patrimonio bibliográfico, sonoro, visual, audiovisual o digital de las culturas de España. Todavía más, hay que añadir otro requisito exigido, como es el de que esos sitios web se encuentren publicados en cualquiera de las lenguas españolas oficiales. $\mathrm{O}$ bien, que hayan sido producidos o editados por cualquier persona física o jurídica, domiciliada o residente en territorio español, así como aquellos productos cuyo dominio esté vinculado a España. Además de cualquier sitio web que albergado en otros dominios, contenga patrimonio documental español. 
Como es lógico, el decreto observa que no hay obligación de depósito para los correos y la correspondencia privada, para los contenidos de una intranet, así como para los ficheros de datos de carácter personal expuestos a un grupo restringido de personas. Por su parte los contenidos dinámicos, generados por recuperaciones de catálogos, bases de datos, repositorios, que no son obtenidos directamente por los robots, también están excluidos de la recogida.

Para el caso de los e-books, conviene diferenciarlos de los sitios web, en tanto se trata de recursos monográficos digitales con características de uso en un entorno electrónico, bien sea de acceso directo o remoto desde un dispositivo (De Beer, et al. 2016). En principio se produce un ligero cambio ya que el proceso pasa por el envío del documento por parte del productor o representante, a una plataforma de distribución ad hoc para que sea realizado el procedimiento de depósito seguro. No se asigna número de DL pero puede tener ISBN u otro número como por ejemplo el DOI en caso de solicitarlo.

Los contenidos editados en la web pueden tener disponibilidad plena de forma que los editores de publicaciones digitales no deben realizar ninguna acción sobre lo que producen, solo han de facilitar la recolección a los sistemas de rastreo de la web, pero los hay que son restringidos, en cuyo caso el productor está obligado a facilitar su captura dando las claves de acceso y reproducción sin que se vean perjudicados los titulares de derechos.

\subsection{Selección para el archivado}

En este escenario no es difícil imaginar que un importante reto reside en qué sitios web se han de capturar. Por una parte, las orientaciones dadas en el reglamento legal son demasiado genéricas y no llegan a especificar una selección precisa de lo que hay que recoger. Por otro lado el volumen de lo publicado diariamente en Internet es demasiado amplio como para pretender guardarlo todo. Es necesario establecer unos criterios específicos que ayuden a los conservadores de documentos en línea a realizar la selección correcta para la captura de los sitios web.

El proceso es laborioso y se ha considerado necesario establecer colaboraciones con instituciones internacionales y con iniciativas nacionales cuya experiencia es de gran ayuda. En el marco del Consejo de Cooperación Bibliotecaria se ha creado un grupo de trabajo denominado "Depósito legal y patrimonio en línea” liderado por bibliotecarios de la BNE y compuesto por una representación de los responsables de conservación de bibliotecas centrales de las comunidades autónomas. Desde 2013 comenzó a reunirse con la intención de colaborar de la manera más eficaz posible en el proceso de archivado de la web en España de modo que el patrimonio digital español quede salvaguardado a largo plazo.

En un principio, en el 2009 la BNE inició una recogida masiva de carácter anual a todas las web del dominio /.es/ ${ }^{3}$. A este archivado hay que añadir una serie de capturas selectivas cuya propuesta de recogida se plantea de varias formas. Se prioriza la elección de los recursos por la relevancia del tema según consideración

3 A fecha de febrero de 2017 se han recogido 1.853.500 registros según la estadística de Dominios.es. 
de los centros conservadores de las comunidades autónomas ${ }^{4}$ y de la BNE. Es esta una tarea complicada, difícil de consensuar por la variedad de intereses locales que se publican.

Son susceptibles de ser integrados en el archivado web aquellos acontecimientos que han tenido repercusión bien por su valor artístico, cultural, científico, social, político para la sociedad española, además de que puedan resultar interesantes a largo plazo desde una perspectiva de investigación. A modo de orientación sirva el interés hacia eventos de tipo político ${ }^{5}$, fallecimiento de personas destacadas, catástrofes y tragedias de carácter humanitario, acontecimientos culturales y deportivos de amplio nivel. Una clasificación general en tres tipos cumple con un intento de distribuir los temas:

1) selección de capturas temáticas que recogen recursos sobre un tema o un tipo de documento, por ejemplo Política catalana o Música y audiovisuales,

2) recolecciones de evento dedicadas a salvaguardar para el futuro acontecimientos de especial relevancia de la sociedad española ${ }^{6}$,

3) las de riesgo buscan recoger los sitios web en peligro de desaparición.

En ese intento de establecer unas características que sirvan de guía aparecen unas prioridades necesarias como por ejemplo las de incluir primeramente aquella información que esté producida únicamente en forma digital, que no tenga un respaldo impreso, priorizando a aquellas web recientemente actualizadas. El carácter objetivo de las capturas debe evitar la valoración de las ideas transmitidas para no primar unas sobre otras.

En cuanto a categorías de recursos, se estiman oportunas las selecciones de medios de comunicación, organismos oficiales, instituciones culturales (archivos, bibliotecas, museos, universidades), asociaciones profesionales, blogs de personas de cierta relevancia (políticos, creadores, investigadores), redes sociales, wikis y videos.

Para conseguir unos criterios precisos es muy importante que las distintas instituciones implicadas colaboren de manera cercana para llegar a un consenso. Ya se ha comentado como positiva la colaboración en el ámbito internacional. A este respecto, la BNE está presente, desde 2010, en el grupo de instituciones que integran el International Internet Preservation Consortium (IIPC). Es reseñable su participación en recolecciones colaborativas con dicha institución como la referida a la crisis humanitaria de los refugiados. Así mismo la cooperación con algunas bibliotecas nacionales que llevan realizando el archivado web sirve como

4 El centro conservador de Galicia recolectó 239 sitios web (144,5 Gb) correspondientes a las Elecciones Autonómicas gallegas. Según el informe sobre 2016 del Grupo de Trabajo, se van poniendo en marcha las colecciones web de las comunidades autónomas sumándose Madrid, Valencia, Murcia, La Rioja, Navarra y Extremadura.

5 Las Elecciones Generales del 20 N de 2011 fueron el tema de la primera recolección selectiva que se realizó, alojándose en los servidores de la BNE a partir de una colaboración entre Internet Archive y Red.es con la BNE. Otra recolección sobre las Elecciones Generales de 2015 arrojó según informe un total de 2130 sitios web alcanzando $14,5 \mathrm{~Tb}$.

6 La abdicación de Juan Carlos I y proclamación de Felipe VI y la muerte de Adolfo Suárez han sido también fruto dentro de las recolecciones de evento selectivas. 
orientación para elegir metodologías o utilizar herramientas ya probadas por alguna de ellas. Como por ejemplo la herramienta NAS (NetArchiveSuite) diseñada desde la Biblioteca Nacional de Dinamarca y utilizada, entre otras, por las de Francia, Austria o Estonia.

\subsection{Gestión del proceso de captura}

La preservación a largo plazo del patrimonio digital empieza por la concepción de sistemas y procedimientos fiables que generen objetos digitales auténticos y estables. (Unesco, 2003).

Lo recogido es intangible, de ahí la dificultad de asegurar la autenticidad y fiabilidad de los documentos. Por ello, los metadatos asociados a las publicaciones también son objeto de captura. La Ley de Transparencia ${ }^{7}$ establece que la información que se publique en sedes electrónicas o páginas web ha de contemplar una serie de mecanismos para facilitar la accesibilidad, la interoperabilidad, la calidad, la reutilización así como la identificación y localización (art. 5.4). Trasladado a las publicaciones en línea que han de ser recogidas por Depósito legal, las webs tendrán como condición el ser identificables por una URL y formarán una unidad documental reconocible (Llueca, 2006). La British Library considera la accesibilidad, la compatibilidad técnica y la permanencia como valores que deben formar parte de la selección de las publicaciones.

Otro reto presentado es el de la noción de territorialidad (De Beer, et al. 2016) al no quedar nítida, ya que la red de la que se capturan los contenidos, excede de las fronteras de un país. Con lo cual, la premisa de recoger lo publicado en un ámbito territorial determinado no funciona para los sitios que se capturan de la web en los que primará para su selección el contenido o la extensión del dominio /.es/. Los autoeditores que publican en la nube, geográficamente independiente, plantean un cambio en el Depósito legal que necesita ser revisado.

La gestión del proceso debe respetar la legislación de propiedad intelectual del mismo modo que se ha venido haciendo con el Depósito legal de las publicaciones tradicionales. Los centros conservadores deben garantizar que no haya descargas, copias o transferencia de ficheros que puedan vulnerar los derechos de autor. Además se garantizará en todo el proceso la protección de datos de carácter personal.

\subsection{Herramientas de gestión}

La infraestructura tecnológica que sirve para gestionar todo el proceso en la BNE se utiliza ya en otras importantes instituciones internacionales, siendo herramientas suficientemente contrastadas. Por ejemplo, Heritrix (heredera) es el robot que rastrea la red, creado por Internet Archive, que se sirve de NetArchiveSuite para guardar todas las webs recogidas según los parámetros de selección preestablecidos. En cada recolección se recorren y se guardan todas las páginas y sitios web. Heritrix es una aplicación de software de código abierto que actúa como

Ley 19/2013 de 9 de diciembre. 
robot de captura pasando por las distintas páginas y sitios web, alojados en los dominios y subdominios programados con antelación. Procede a recolectar los elementos que componen el site (texto, ficheros de audio, imágenes, vídeos, etc.), para a continuación comprimir las versiones resultantes en un formato que permita su almacenamiento. En este caso se archivan en formato WARC, norma ISO 28500:2009. Las recolecciones pretenden la reproducción fiel del aspecto del sitio durante la captura, de modo que la réplica tenga las mismas funcionalidades de la versión original.

La herramienta para organizar los contenidos de acuerdo a unos parámetros concretos es Cweb. Permite crear, gestionar y describir las colecciones de sitios web. Se trata de una herramienta desarrollada por la Biblioteca Nacional Francesa y ofrecida por la BNE a los centros de conservación de las comunidades autónomas a través de la red SARA ${ }^{8}$ de la Administración General del Estado.

Las instituciones de custodia necesitan organizar lo recogido utilizando las clasificaciones que permiten la recuperación. La asignación de materias y palabras clave necesarias para indizar el contenido y asegurar una eficaz recuperación de las colecciones se basa en criterios estrictamente bibliotecarios bajo las normas del Manual y del Catálogo de Autoridades de la BNE así como del Manual de Indización de Encabezamientos de Materia y la CDU. La posibilidad de acceso a lo recopilado la ofrece OpenWayback con interfaz de búsqueda simple o avanzada como cualquier interfaz común.

\subsection{Acceso y recuperación}

Hay que disponer de una interfaz de acceso a lo archivado y a lo recogido como Depósito legal electrónico que deberá consultarse en terminales instalados en salas de la BNE o en los centros de conservación de las comunidades autónomas.

El usuario puede realizar el acceso a ese repositorio documental hoy por hoy desde ordenadores que estén bloqueados (offline) y solo sirvan como mero depósito para aquellos documentos que tengan restricciones de derechos de propiedad intelectual. Motivos que obligan a ello derivan de la protección de los derechos de propiedad intelectual. Los editores deben facilitar los medios para acceder a publicaciones sujetas a DRM (Digital Rights Management). Mediante la herramienta OpenWayback se permite la búsqueda por URL, pero es necesario ofrecer el acceso por colecciones, materias o incluso por texto completo.

\section{Modelos en otros países}

En el ámbito internacional hay acciones de archivado de la web que llevan realizándose desde hace tiempo y han servido de orientación a la hora de establecer una metodología que pueda resultar útil para aplicarla en el caso de España. Uno de

8 Sistema de Aplicaciones y Redes para las Administraciones. 
los principales retos está en decidir qué es lo que se va a recoger para la conservación a largo plazo. De tal modo que el método para la captura tiene como base dos modelos completamente opuestos.

Por un lado el Proyecto Kulturarw3, desarrollado en la Biblioteca Nacional de Suecia, basa su procedimiento en una recogida exhaustiva e integral realizada por un motor rastreador de la red, procediendo a capturar todos aquellos registros que identifica bajo el dominio sueco, indexando y guardando copia de los mismos. La Biblioteca Nacional Sueca recoge sitios web desde 1997. Este modelo lo han seguido en Noruega, Finlandia, Islandia y Austria, entre otros (Llueca, 2005).

En el lado opuesto se encuentra la Biblioteca Nacional de Australia quien, con el Proyecto Pandora, opta por un modelo selectivo de recogida que garantiza una mayor coherencia en la colección de recursos ya que son seleccionados previamente, si bien es cierto, esta manera de proceder ofrece una visión sesgada del patrimonio australiano (Serra, 2006; Cordón, 2006). Canadá, Japón y Reino Unido siguen este modelo. Por ejemplo, Canadá ha recogido desde 2005 contenido web federal y no federal. Éste último archivado se nutre de informaciones tales como las elecciones federales, o cuestiones relacionadas con el Primer Centenario de la Guerra Mundial, o la Comisión de la Verdad y la Reconciliación o las Olimpiadas de Sochi de 2014. Actualmente el contenido alcanza más de 5 petabytes de material digital si se añade la colección digitalizada del material impreso. Su política de preservación a largo plazo está regida por principios confiables, colaborativos y sostenibles que ofrecen una garantía ante vaivenes tecnológicos permanentes. La Biblioteca Nacional de Alemania recoge de manera exhaustiva, desde 2010, aproximadamente unas 300 cabeceras de prensa diaria, permitiendo el acceso a la misma desde las salas de lectura habilitadas para ello en las bibliotecas de Leipzig y Frankfurt con un periodo de retardo de 8 días. La captura de sitios web se ha iniciado en 2012 valorando la participación externa para su realización. Además, la colaboración con instituciones académicas permite que la Biblioteca Nacional Germana recoja, catalogue y conserve tesis doctorales y trabajos de investigación. Mediante el uso del formato XMetaDissPlus, implementado para distribuir los metadatos de tesis doctorales, en el marco del Proyecto Electronic Dissertations Plus (eDissPlus) entre la Universidad Humboldt de Berlín y la Biblioteca Nacional, permite disponer de datos e informes de investigación con garantía de acceso y recuperación, tal y como preconizan las políticas Open Access.

La evolución de estos procedimientos ha empujado a un cambio con respecto al comienzo del proceso de archivado. Así, países que inicialmente seguían un modelo integral, poco a poco han ido capturando recursos selectivos significativos. Los desarrollos del proceso de archivado web en el inicio se decantaban de manera única por un modelo u otro, a medida que se ha ido avanzando, la tendencia es a conjugar ambos, bien sea porque se han identificado las ventajas y los inconvenientes de ambos, o bien porque las instituciones se han animado a cooperar. Un ejemplo de esto son las soluciones adoptadas en países como Francia, Dinamarca y Nueva Zelanda. Tal y como vaticinó Llueca (2005), el 
modelo del futuro será el híbrido, ya no habrá lugar para que exista una diferencia categórica a la hora de proceder.

En el caso de España, el modelo híbrido es el aceptado mayoritariamente, porque permite combinar capturas exhaustivas de sitios web con otras selectivas definidas a partir de criterios previamente establecidos de modo que permite un mayor horizonte de contenidos a conservar.

Las capturas exhaustivas recogen de manera amplia todas aquellas URL que muestren genuinamente aspectos de la imagen representativa del país. Principalmente las web que tienen el dominio /.es/, o bien están en otros dominios pero contienen patrimonio documental español, o las que están realizadas en cualquiera de las lenguas oficiales de España.

Las selectivas responden a criterios que tienen que ver con una temática representativa de algún asunto susceptible de ser conservado, o bien relacionado con acontecimientos relevantes de carácter cultural, o con la creación artística, o la ciencia, o la política o la sociedad, o también sitios web que vayan a ser clausurados y exista interés por conservarlos. Los niveles de profundidad en la captura de cada sitio web (semillas ${ }^{9}$ ) deberán de establecerse de manera singular conociendo el grado de interés o representatividad que ofrecen. En general las capturas masivas no alcanzan el nivel de profundidad que tiene el de las selectivas, ya que estas últimas responden al objetivo de completar la información de acuerdo a su relevancia.

\section{Los conservadores de la web en España}

Los países desarrollados están convencidos de la necesidad de preservar su patrimonio digital. (Llueca, 2005). Y lícitamente corresponde a las bibliotecas, los archivos y los museos realizar esta tarea (Ramos, 2013). En España el proceso de gestión, identificación y preservación a largo plazo de las publicaciones en línea lo tutelan los bibliotecarios del Área de Gestión del Depósito de las Publicaciones en Línea de la BNE en colaboración con los centros de conservación de las comunidades autónomas encargados del archivado web. Sin embargo existen dos iniciativas en España donde se viene realizando la preservación de documentos en línea desde hace tiempo. Por un lado la Biblioteca de Cataluña lleva a cabo el proyecto PADICAT ${ }^{10}$ desde 2005 que compila, procesa y da acceso a la producción digital de Cataluña y en el País Vasco, de manera similar, se ha desarrollado el proyecto ONDARENET ${ }^{11}$ desde 2007.

Al igual que la dificultad de realizar una selección de materiales para integrarlos en la colección de una biblioteca, los profesionales conservadores que realizan el proceso de gestión de los sitios web han de ser muy versados para identificar

9 Se denomina semilla a la URL de la que se parte para realizar una recolección. Puede coincidir con la página principal de un sitio web o bien ser una sección o un documento que esté incluido en un sitio web y que incluso tenga un formato diferente (mp3, avi, mobi, e-pub).

10 Disponible en http://www.padicat.cat/es

11 Disponible en http://www.ondarenet.kultura.ejgv.euskadi.eus:8085/ondarenet/ 
aquellos recursos en línea que deben integrarse para su conservación futura como Depósito legal.

Esa necesidad de llegar a un acuerdo que normalice los criterios de selección queda patente por la dificultad que entraña discernir ante una diversidad de producciones.

Salvo estos proyectos que existen en Cataluña y País Vasco, en el resto de España existen bibliotecas digitales que en mayor o menor grado recogen digitalizaciones realizadas sobre el patrimonio que custodian algunas de las bibliotecas de su comunidad autónoma ${ }^{12}$, organizadas bajo estándares de interoperabilidad válidos para intercambiar archivos, pero no se asemeja al archivado de la web que lleva otro camino.

En relación con la predisposición de los profesionales ante todos estos cambios, Hockx-Yu citado por De Beer et al. (2016), considera que se requieren nuevos flujos de trabajo, nuevas herramientas, pero sobre todo, establecer relaciones cooperativas con profesionales de áreas tecnológicas. En suma, este autor piensa que la estructura de la organización que soporte esto todavía se está diseñando.

Entretanto, cabe la posibilidad de que amplias colecciones procedentes de grandes repositorios documentales, como por ejemplo centros universitarios o plataformas de libros electrónicos, ofrezcan garantías de preservación y acceso a largo plazo sin que tengan que transferir los contenidos de sus repositorios, habilitando enlaces a los contenidos, eso sí, bajo convenios de colaboración previamente establecidos.

Sirva como dato que durante el 2016 se ha ampliado el alcance de la recolección diaria de prensa, radio y televisión, que ha pasado de 29 a 90 semillas. De forma selectiva se recolectó el evento relacionado con las conmemoraciones en torno a Cervantes. En total las recolecciones abarcan unos 72 TB de información en formato WARC y más de 300 millones de URL. ${ }^{13}$

Cabe comentar aquí la iniciativa que promueve el servicio Archive-It (filial de Internet Archive) junto a 13 instituciones piloto norteamericanas y canadienses, que han implementado lo que denominan el Modelo de Ciclo de Vida del Archivado web para promover buenas prácticas en todas las fases por las que pasa una institución en la gestión del archivado web. Desde los objetivos del programa pasando por políticas de acceso, reutilización así como conservación y gestión de riesgos, lo que se persigue es un marco que unifique el proceso. Cada paso del ciclo se desglosa de manera individual aunque integrado en diversas fases, mostrándolo gráficamente a modo de círculos concéntricos (Hanna, 2014).

12 Biblioteca Virtual de Andalucía en http://bibliotecavirtualdeandalucia.es , Biblioteca Virtual de Aragón (Biviar) en http://bibliotecavirtual.aragon.es , Memoria Digital de Canarias (mdC) en http://mdc.ulpgc.es , Biblioteca Digital de Castilla y León (BDCYL) en http://bibliotecadigital.jcyl.es , Ciconia. Biblioteca Digital del Patrimonio Cultural de Extremadura en http://ciconia.gobex.es , Galiciana- Biblioteca Digital de Galicia en http://biblioteca.galiciana.gal , Biblioteca Digital de la Comunidad de Madrid en http://www.bibliotecavirtualmadrid.org

13 Datos extraídos del Informe del Grupo de Trabajo de Depósito Legal y Patrimonio Digital, 2017. 
No obstante, se debe asegurar la autenticidad y confiar en que el proceso de recuperación y acceso conserva todos los derechos de propiedad intelectual así como la autenticidad real del documento.

\section{Conclusiones}

La implementación del proceso de Depósito legal electrónico en España se ve favorecida por la colaboración con distintas instituciones de ámbito internacional que han encontrado y solucionado problemas con anterioridad.

Las recolecciones de tipo mixto intentarán solventar, en la medida de lo posible, el hándicap que supone abarcar todo el panorama de lo que se publica de forma electrónica en cada momento, pero creemos que el mayor reto reside en establecer unos criterios de selección para realizar las capturas de los sitios web sin que supongan un menoscabo en la memoria patrimonial a largo plazo.

La posibilidad que existe de almacenar entre varias instituciones, ejemplo el caso de España, entre la BNE y las comunidades autónomas y las Universidades, permite un desarrollo de captura mixto en el que se distribuye la recogida, de modo que es el ámbito territorial el que puede imponer la captura de lo local, tal como cabeceras de prensa y webs locales.

Todo ello se realiza para preservar la memoria de este tiempo, expresada de forma intangible, dotada de suma fragilidad y que las instituciones de la memoria se ven obligadas a custodiar para las generaciones futuras.

\section{Referencias bibliográficas}

Arco Blanco, A. del ; Rojas Sosa, L. (2016). El archivo de internet: Depósito legal de las publicaciones electrónicas tras el Real Decreto 635/2015, de 10 de junio. Granada: Comares. Recuperado de: https://dialnet.unirioja.es/descarga/libro/655559.pdf [consulta: 03/03/2017].

Cordón García, J.A. (2006). El depósito legal y los recursos digitales en línea. En: Las Bibliotecas Nacionales del Siglo XXI. Valencia: Biblioteca Valenciana. 97-114.

Davara Rodríguez, M. A. (2015). El depósito legal de las publicaciones en línea. Consultor de los ayuntamientos y de los juzgados: Revista técnica especializada en administración local y justicia municipal, (19), 2356-2367.

De Beer, M., Van der Merwe, M., Ball, L., Fourie, I. (2016). Legal deposit of electronic books - a review of challenges faced by national libraries. Library Hi Tech, 34(1), 87103.

España. Real Decreto 635/2015 de 10 de julio, por el que se regula el depósito legal de las publicaciones en línea. Boletín Oficial del estado, 25 de julio de 2015, núm. 177, pp. 62878-62885.

España. Ley Orgánica 19/2013 de 9 de diciembre, de transparencia, acceso a la información pública y buen gobierno. Boletín Oficial del estado, 10 de diciembre de 2013, núm. 295, pp. 97922-97952.

España. Ley Orgánica 23/2011, de 29 de julio de depósito Legal. Boletín Oficial del estado, 30 de julio de 2011, núm. 182, pp. 86716-86727. 
Gompel, R. y Svensson, L. (2011). La gestión del Depósito Legal de las publicaciones en línea en Alemania. 77th IFLA General Conference and Assembly. Libraries beyond libraries: Integration, Innovation and Information for all. Recuperado de: http://conference.ifla.org/ifla77. [consulta: 05/02/2018].

Hanna, K. (2014). El Modelo de Ciclo de Vida del Archivado Web. Anuario AC/E de Cultura Digital.

Jornada sobre Archivos Web y D.L. Electrónico. Biblioteca Nacional de España. (9-72013). Recuperado de: https://www.youtube.com/watch?v=K5lZTtfX1cs (consulta: 20/05/2017).

Llueca, C. (2005). Webs siempre accesibles: las bibliotecas nacionales y los depósitos digitales nacionales. BiD: Textos universitaris de biblioteconomia i documentació, (15), 3. Recuperado a partir de http://diposit.ub.edu/dspace/bitstream/2445/8953/1/ 548444esp.pdf [consulta: 11/04/2017].

Llueca, C.. (2006). Archivando la Web, el proyecto Padicat (Patrimonio Digital de Cataluña). El profesional de la información, 15(6), 473-478. http://doi.org/10.3145/epi.2006.nov.09. [consulta: 11/04/2017].

Pulgar, F., Modesto, V., \& Tamayo, M. (2013). La captura de la web vasca: Un caso práctico de preservación digital. Preservar, 22-24.

Ramos Simón, F. (2013). Lo viejo y lo nuevo: el patrimonio cultural digitalizado. Preguntas de investigación. VI Jornada Profesional de la Red de Bibliotecas del Instituto Cervantes. La investigación española en documentación: ¿en qué liga jugamos? (6 ${ }^{\mathrm{a}}$. Madrid). Recuperado de: www.cervantes.es/imagenes/File/ ponencia_fernando_ramos_rbic.pdf [consulta: 05/02/2018].

Serra Aranda, E. (2006). Archivando la Web catalana : iniciativas cooperativas de Qué se está haciendo al respecto: una aproximación a la situación a escala internacional. En $L a$ recuperación de la memoria, muchas más oportunidades que realidades: el trabajo cooperativo de archivos, bibliotecas y museos. Universidad del País Vasco, 23-25 agosto.

Unesco. Carta sobre la preservación del patrimonio digital. 15-10-2003. Recuperado de: .http://portal.unesco.org/es/ev.php-URL_ID=17721\&URL_DO=DO_TOPIC\&URL_ SECTION=201.html [consulta: 13/12/2016]. 